\title{
Transatlantic editorial: A comparison between European and North American guidelines on myocardial revascularization
}

\author{
Philippe Kolh, MD, PhD, ${ }^{\mathrm{a}}$ Paul Kurlansky, MD, ${ }^{\mathrm{b}}$ Jochen Cremer, MD, PhD, ${ }^{\mathrm{c}}$ Jennifer Lawton, MD, \\ Matthias Siepe, MD, ${ }^{\mathrm{e}}$ and Stephen Fremes, $\mathrm{MD}, \mathrm{MSc}^{\mathrm{f}}$
}

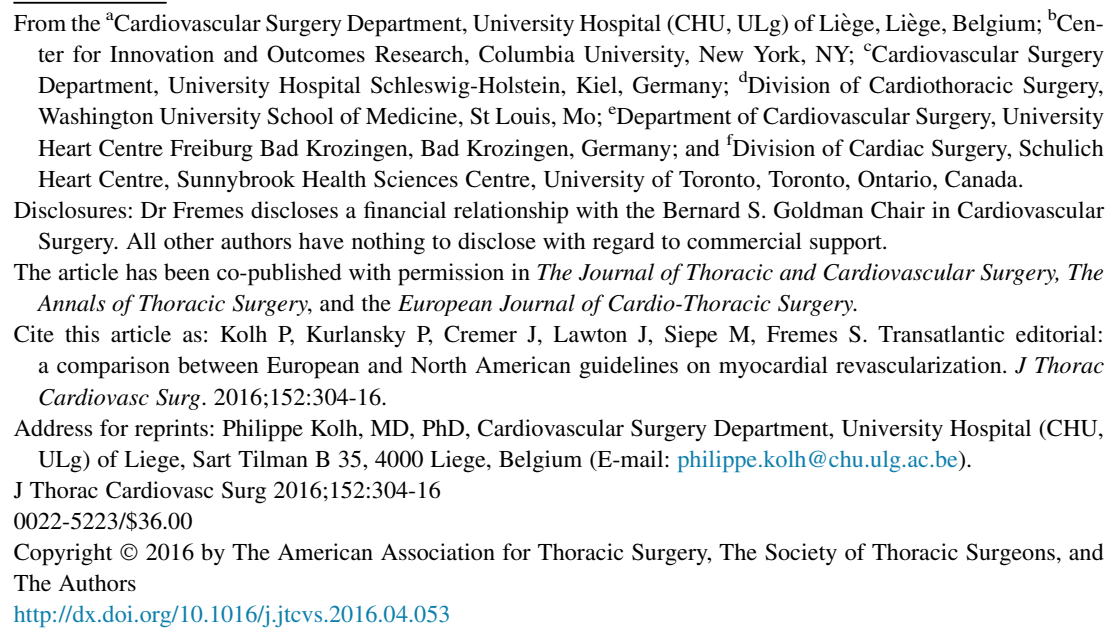

The 2014 edition of the European Association for Cardio-Thoracic Surgery (EACTS) and the European Society of Cardiology (ESC) joint Guidelines on Myocardial Revascularization (MR) marks the 50th anniversary of the first coronary artery bypass grafting $(\mathrm{CABG})$ procedure. The first percutaneous coronary intervention $(\mathrm{PCI})$ procedure was performed 13 years later, in 1977. Since these early times, MR techniques have gained clinical importance worldwide and are now one of the most commonly performed interventions in modern medicine. On the other side of the Atlantic, the American societies have also published several guidelines on MR: In 2011, the ACCF/AHA Guidelines for CABG Surgery $^{2}$; the 2012 ACCF/AHA/ACP/AATS/PCNA/ SCAI/STS guideline for the diagnosis and management of patients with stable ischemic heart disease ${ }^{3}$; the 2014 ACC/AHA/AATS/PCNA/SCAI/STS focused update ${ }^{4}$ and in 2015, the Society of Thoracic Surgeons (STS) Clinical Practice Guidelines on Arterial Conduits.

In view of the rapidly evolving landscape of therapeutic options, this Transatlantic Editorial is intended to compare the European and American societies' guidelines on MR, covering important topics such as decision-making, patient information, timing of revascularization, risk scores, ischemia testing, revascularization with $\mathrm{CABG}$ versus PCI, use of arterial conduits in CABG, on-pump versus off-pump surgery, revascularization in diabetic patients and implementation of guidelines.

\section{DECISION-MAKING AND HEART TEAM}

The American as well as European guidelines strongly advocate the implementation of "Heart Team" decisions

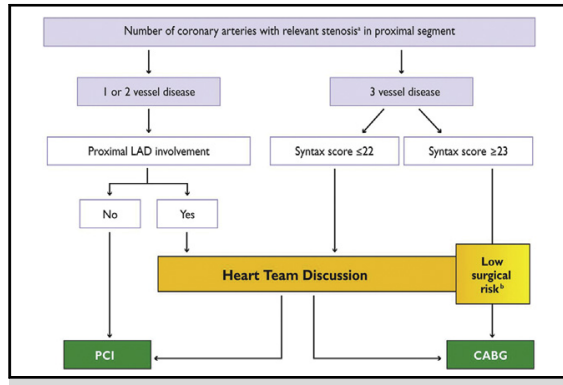

A treatment algorithm for $\mathrm{PCl}$ or $\mathrm{CABG}$ in stable $\mathrm{CAD}$ without LM involvement.

\section{Central Message}

This editorial compares myocardial revascularization guidelines between North America and Europe. Based on the differences, new recommendations are made to improve patient outcomes. for complex and stable coronary artery disease (CAD) as a class of recommendation (COR) I, with level of evidence (LOE) C. Recommendations for Heart Team involvement in stable multivessel CAD are stronger in the European guidelines ("required") compared with the American guidelines ("recommended"). Furthermore, the description of the Heart Team differs: According to the EACTS/ESC Guidelines, at least three specialists (clinical cardiologist, interventional cardiologist and surgeon) should meet on a regular basis and protocols be followed. ${ }^{1}$ On the other side of the Atlantic, the American guidelines do not describe this multidisciplinary Heart Team in a conference style, but recommend that the interventional cardiologist and surgeon, together as a Heart Team, should discuss the treatment options. ${ }^{3}$ For centres without infrastructure for on-site coronary surgery, the European guidelines recommend institutional protocols that need to be established with partner institutions providing surgery.

The benefit of a Heart Team decision is convincingly presented throughout all available literature in line with the authors' attitudes. The superiority of a team decision-based treatment is derived from comparing randomized and registry cohorts with better results for the registry cohorts. ${ }^{6,7}$ It has been shown that the initiation of the structured Heart Team approach could lead to beneficial clinical outcomes. ${ }^{8}$ Other centres report that the decision and referral strategies did not change at all after initiation of the European 
guidelines, which is a clear example of how deep-set local habits and beliefs can be and how resistant some practitioners can be to change. ${ }^{9}$ Interestingly, re-discussing the same patients after 1 year leads to different decisions in $24 \%$ of the cases. This fact underscores that, in some CAD patients, both treatment modalities might be appropriate. ${ }^{10}$ Also, the importance of including other clinical specialists as part of the Heart Team is reflected by the fact that taking the severe cases into this conference might lead to a significant proportion of treatment recommendations other than MR (eg, heart transplantation, ventricular assist device, valve surgery or medical therapy). ${ }^{8}$

\section{PATIENT INFORMATION}

Patient consent discussion is handled differently in the existing guidelines. While the EACTS/ESC Guidelines expand on that topic including specific recommendation categorization, informed consent is only mentioned as a prerequisite of "any invasive or non-invasive procedure" in the American guidelines. ${ }^{3}$ Conversely, the American guidelines are much more precise on the topic of Patient Education.

The EACTS/ESC Guidelines put forward the importance of patient information and need for an extensive informed consent process. They conclude that enough time should be allowed for informed decision-making. Specifically, in a high proportion of patients with stable CAD, a gap between diagnostic angiography and revascularization should exist to allow sufficient time to receive information about all therapeutic alternatives. Written informed consent is specifically needed for all procedures done with the exception of patients in shock or with ST-segment elevation myocardial infarction (STEMI).

\section{TIMING}

The treatment of STEMI patients with primary emergency $\mathrm{PCI}$ is unquestionable. The EACTS and ESC representatives have included this patient cohort in the joint guidelines, whereas the major American societies have formulated separate guidelines for the management of STEMI. ${ }^{11}$ For those patients with non-ST-segment elevation (NSTE)-acute coronary syndrome (ACS), the European guidelines recommend revascularization within 24 or $72 \mathrm{~h}$, according to patient risk stratification. Primary criteria for urgency (invasive strategy within $24 \mathrm{~h}$ ) are met with rising troponin levels, dynamic ST-segment or T-wave changes or a GRACE score of $>140 .{ }^{1}$ In the American guidelines, ${ }^{2-4}$ the recommendation for these urgent patients is based on a more general rule, indicating that the acuity of presentation and extent of ischemia dictate the timing of intervention. Interestingly, both guidelines see only the need for revascularization strengthened. However, the choice of revascularization method is mainly independent of the urgency and influenced by the same considerations for choosing PCI or CABG in the stable patient cohort. However, American guidelines state that PCI is reasonable in patients undergoing revascularization for NSTE-ACS. Both guidelines favor CABG over PCI for NSTE-ACS patients with diabetes mellitus with complex CAD.

Comparisons between American and European Guidelines for patients with NSTE-ACS are outlined in Table 1.

For stable patients without severe symptoms, the EACTS/ ESC Guidelines consider a maximum waiting time of 6 weeks to revascularization appropriate. Whenever symptoms are severe, anatomy high-risk or left ventricular function depressed, the European guidelines recommend revascularization within 2 weeks. ${ }^{1}$ Interestingly, the American guidelines do not cover this problem. The waiting times in American centres appear to have been reduced and this topic might not be of the same significance as in previous years. ${ }^{14}$ Also, differences in payer systems in Europe and America contribute to timing considerations. In addition, cultural and social expectations are likely to be different among patients and cardiologists across the Atlantic.

\section{RISK SCORES}

Various risk scores validated for the short-term mortality after CABG are available (STS score, EuroSCORE and EuroSCORE II, ACEF), but these scores do not predict medium- or long-term outcome. The SYNTAX score was developed to summarize the complexity of coronary lesions. ${ }^{15}$ It was found that medium- and long-term outcomes correlated with the SYNTAX score. Both guidelines see an important role of using risk scores-especially the SYNTAX score. The American guidelines provide a COR IIa (LOE B) for the use of STS and SYNTAX scores in patients with complex CAD and unprotected left main (LM) disease, whereas the EACTS/ESC Guidelines recommend the use of the SYNTAX score to assess medium- to longterm outcome before CABG or PCI (COR I, LOE B). The STS score (COR I, LOE B) or the EuroSCORE II (COR IIa, LOE B) should be used to assess short-term outcome after CABG. Also, some recommendations in the choice of treatment modality are based on the SYNTAX score in the guidelines (see specific paragraph). Of note, limitations exist in all risk models and the performance in the specific centre's patient cohort should be taken into consideration. The risk scores should only be used as an adjunct, whereas the Heart Team's decision based on the clinical profile remains essential.

\section{ISCHEMIA TESTING}

The EACTS/ESC Guidelines recommend diagnostic testing in stable CAD only in symptomatic patients and based on the probability of significant disease. In patients with intermediate probability $(15 \%-85 \%)$ of significant disease, functional testing using stress echocardiography, nuclear imaging, stress MRI or PET perfusion scan is 
TABLE 1. ACCF/AHA and EACTS/ESC Guidelines on NSTEMI

\begin{tabular}{|c|c|c|c|c|}
\hline & $\begin{array}{c}\text { ACCF/AHA } 2011\left(\text { Hillis }^{2}\right) \\
\text { and } 2012\left(\text { Fihn }^{3}\right)\end{array}$ & $\begin{array}{c}\text { ACC/AHA } 2014 \text { Focused } \\
\text { Update (Amsterdam }{ }^{12} \text { ) }\end{array}$ & ESC $2011\left(\right.$ Hamm $\left.^{13}\right)$ & EACTS/ESC $2014\left(\right.$ Kolh $\left.^{1}\right)$ \\
\hline \multirow[t]{2}{*}{ NSTEMI } & $\begin{array}{l}\text { PCI vs CABG reference previous } \\
\text { published guidelines for } \\
\text { revascularization in stable } \\
\text { CAD }\end{array}$ & & & $\begin{array}{l}\text { Class I: PCI vs CABG with } \\
\text { multivessel disease or complex } \\
\text { lesions should be discussed } \\
\text { with Heart Team }\end{array}$ \\
\hline & & $\begin{array}{l}\text { Class IIb (LOE B). A strategy of } \\
\text { multivessel PCI, in contrast to } \\
\text { culprit-only PCI, may be } \\
\text { reasonable in patients } \\
\text { undergoing coronary } \\
\text { revascularization as part of } \\
\text { treatment for NSTE-ACS }\end{array}$ & $\begin{array}{l}\text { Class I (LOE C). The } \\
\text { revascularization strategy } \\
\text { (ad hoc culprit lesion PCI/ } \\
\text { multivessel PCI/CABG) should } \\
\text { be based on the clinical status } \\
\text { as well as the disease severity } \\
\text { (SYNTAX score), according to } \\
\text { the Heart Team protocol }\end{array}$ & $\begin{array}{l}\text { Class I (LOE C). It is } \\
\text { recommended to base } \\
\text { revascularization strategy } \\
\text { (ad hoc culprit lesion PCI } \\
\text { multivessel PCI/CABG) on } \\
\text { clinical status and } \\
\text { comorbidities as well as disease } \\
\text { severity (SYNTAX) according } \\
\text { to the Heart Team protocol }\end{array}$ \\
\hline $\begin{array}{l}\text { Diabetic } \\
\text { patients }\end{array}$ & & $\begin{array}{l}\text { Class IIa (LOE B). Reasonable to } \\
\text { choose CABG over PCI in } \\
\text { older ( } \geq 75 \text { years) patients with } \\
\text { NSTE-ACS who are } \\
\text { appropriate candidates, DM, } \\
\text { three-vessel CAD (SYNTAX } \\
\text { >22) with or without } \\
\text { involvement of proximal LAD } \\
\text { to decrease events and } \\
\text { readmission, and improve } \\
\text { survival }\end{array}$ & $\begin{array}{l}\text { Class I (LOE B). CABG should be } \\
\text { favored over PCI in diabetic } \\
\text { patients with main stem lesions } \\
\text { and/or advanced multivessel } \\
\text { disease }\end{array}$ & \\
\hline $\begin{array}{l}\text { Unprotected } \\
\text { LM }\end{array}$ & $\begin{array}{l}\text { Class IIa (LOE B) PCI for } \\
\text { unstable angina/NSTEMI if not } \\
\text { a CABG candidate }\end{array}$ & & & $\begin{array}{l}\text { Class I (LOE C). Recommend to } \\
\text { base revascularization strategy } \\
\text { on clinical status and } \\
\text { comorbidities as well as the } \\
\text { disease severity (SYNTAX) } \\
\text { according to the local Heart } \\
\text { Team protocol }\end{array}$ \\
\hline
\end{tabular}

$\overline{A C C F}$, American College of Cardiology Foundation; $A C S$, acute coronary syndrome; $A H A$, American Heart Association; $C A B G$, coronary artery bypass grafting; $C A D$, coronary artery disease; $D M$, diabetes mellitus; $E A C T S$, European Association for Cardio-Thoracic Surgery; ESC, European Society of Cardiology; $L A D$, left anterior descending; $L M$, left main; LOE, level of evidence; NSTEMI, non-ST-segment elevation myocardial infarction; PCI, percutaneous coronary intervention.

recommended (COR I, LOE A for all four modalities), while CT angiography should be considered (COR IIa, LOE A). In case of higher probability, coronary angiography is recommended (COR I, LOE A). Exercise electrocardiogram (ECG) is not mentioned.

The American guidelines delve into the recommendation of diagnostic tests in greater detail, which are presented in an algorithm. ${ }^{3}$ Taking together the COR I from the algorithm, they recommend the use of exercise ECG in those patients with interpretable ECG (COR I, LOE A). In patients with non-interpretable ECG, exercise test with nuclear imaging or echocardiography is advocated (COR I, LOE B). In patients unable to exercise, pharmacological stress nuclear imaging or echocardiography should be performed (COR I, LOE B). CT angiography should be considered (COR IIa, LOE C) in several circumstances with inconclusive results or inability to perform class I recommended tests.

\section{MYOCARDIAL REVASCULARIZATION WITH CABG VERSUS PCI}

With the 5-year results of the SYNTAX trial showing a clear survival benefit for several surgical subgroups, ${ }^{16}$ the European guidelines focus very precisely on the grading of the complexity of the coronary disease according to the original SYNTAX score. Thus, these guidelines give a clear COR I (LOE A or B) for surgery of any coronary disease exhibiting proximal left anterior descending (LAD) coronary artery stenosis, any three-vessel disease and any LM stenosis. However, PCI is recommended as an alternative for patients with one- and two-vessel disease with proximal LAD involvement, LM disease with a low SYNTAX score and three-vessel disease also with a low SYNTAX score. Conversely, PCI should not be used (COR III) in patients with LM disease and high SYNTAX score or with threevessel disease and intermediate or high SYNTAX score. 
TABLE 2. ACCF/AHA and EACTS/ESC Guidelines on Myocardial Revascularization

Guidelines for myocardial revascularization to improve survival in stable ischemic disease

$\begin{array}{cc}\text { ACCF/AHA } 2011\left(\text { Hillis }^{2}\right) & \text { ACC/AHA } 2014 \\ \text { and } 2012\left(\text { Fihn }^{3}\right) & \text { Focused Update }\left(\text { Fihn }^{4}\right)\end{array}$
$\begin{array}{cc}\text { Unprotected LM or } & \text { Class } 1 \text { (LOE C): Heart Team approach } \\ \text { complex CAD } & \text { recommended } \\ \text { Class IIa (LOE B): calculation of STS and } \\ \text { SYNTAX score }\end{array}$

Unprotected LM

Three-vessel CAD with Class I (LOE B): CABG

or without proximal

LAD disease

- Class IIa (LOE B): CABG reasonable over PCI with complex three-vessel CAD (SYNTAX $>22$ ) who are good candidates for $\mathrm{CABG}$

- Class IIb (LOE B): PCI of uncertain benefit

Two-vessel CAD with proximal LAD disease
Class I (LOE B): CABG recommended

Class IIa (LOE B): PCI for stable ischemic heart disease when both: Low-risk PCI procedura haracteristics predict significantly mortality $\geq 5 \%$ )

Class IIb (LOE B): PCI when both: Anatomical conditions associated with (SYNTAX $<33$, bifurcation LM CAD) and clinical characteristics that predict increased risk of adverse surgical outcomes (moderate-severe COPD, disability from prior stroke, prior cardiac surgery) (STS predicted operative mortality $>2 \%$ )

Class III (LOE B): Harm when PCI chosen in patients with unfavorable anatomy for PCI and who are good candidates for $\mathrm{CABG}$

\section{EACTS/ESC $2014\left(\right.$ Kolh $\left.^{1}\right)$}

Multidisciplinary decision-making required for multivessel stable $\mathrm{CAD}$

Class I (LOE C). Institutional protocols are developed by the Heart Team to implement appropriate revascularization strategy in accordance with guidelines

Class I (LOE B): CABG for LM and SYNTAX $\leq 22$

Class I (LOE B) for PCI

SYNTAX 23-32

Class I (LOE B): CABG

Class IIa (LOE B): PCI
SYNTAX $>32$

Class I (LOE B): CABG

Class III (LOE B): Harm PCI

SYNTAX $\leq 22$

Class I (LOE A): CABG

Class I (LOE B): PCI

SYNTAX 23-32

Class I (LOE A): CABG

Class III (LOE B): Harm PCI

\section{SYNTAX $>32$}

Class I (LOE A): CABG

Class III (LOE B): Harm PCI

Class I (LOE B): CABG

Class I (LOE C): PCI 
TABLE 2. Continued

\begin{tabular}{|c|c|c|c|}
\hline & $\begin{array}{l}\text { ACCF/AHA } 2011\left(\text { Hillis }^{2}\right) \\
\text { and } 2012\left(\text { Fihn }^{3}\right)\end{array}$ & $\begin{array}{c}\text { ACC/AHA } 2014 \\
\text { Focused Update }\left(\text { Fihn }^{4}\right)\end{array}$ & EACTS/ESC $2014\left(\right.$ Kolh $\left.^{1}\right)$ \\
\hline \multirow[t]{2}{*}{$\begin{array}{l}\text { Two-vessel CAD } \\
\text { without proximal } \\
\text { LAD }\end{array}$} & $\begin{array}{l}\text { Class IIa (LOE B): CABG with extensive } \\
\text { ischemia } \\
\text { Class IIb (LOE C): CABG of uncertain } \\
\text { benefit without extensive ischemia }\end{array}$ & & Class IIb (LOE C): CABG \\
\hline & $\begin{array}{l}\text { Class IIb (LOE B): PCI of uncertain } \\
\text { benefit }\end{array}$ & & Class I (LOE C): PCI \\
\hline \multirow[t]{2}{*}{$\begin{array}{l}\text { One-vessel proximal } \\
\text { LAD }\end{array}$} & $\begin{array}{l}\text { Class IIa (LOE B): CABG with LIMA for } \\
\text { long-term benefit }\end{array}$ & & Class I (LOE A): CABG \\
\hline & Class IIb (LOE B): PCI uncertain benefit & & Class I (LOE A): PCI \\
\hline \multirow[t]{2}{*}{$\begin{array}{l}\text { One-vessel without } \\
\text { proximal LAD }\end{array}$} & $\begin{array}{l}\text { Class III (LOE B): Harm when CABG } \\
\text { chosen }\end{array}$ & & Class IIb (LOE C): CABG \\
\hline & $\begin{array}{l}\text { Class III (LOE B): Harm when PCI } \\
\text { chosen }\end{array}$ & & Class I (LOE C): PCI \\
\hline Diabetic patients & $\begin{array}{l}\text { Class IIa (LOE B): CABG reasonable } \\
\text { over PCI to improve survival in } \\
\text { patients with multivessel CAD and } \\
\text { DM, particularly if LIMA used to LAD }\end{array}$ & $\begin{array}{l}\text { Class I (LOE B): CABG is generally } \\
\text { preferred vs PCI to improve survival in } \\
\text { patients with DM and multivessel CAD } \\
\text { for which revascularization is likely to } \\
\text { improve survival (three-vessel CAD or } \\
\text { complex two vessel CAD involving } \\
\text { proximal LAD), particularly if LIMA } \\
\text { can be anastomosed to LAD, provided } \\
\text { a good candidate for surgery } \\
\text { Class I (LOE C): A Heart Team approach } \\
\text { to revascularization is recommended in } \\
\text { patients with DM and complex } \\
\text { multivessel CAD }\end{array}$ & $\begin{array}{l}\text { Class I (LOE A): Patients with } \\
\text { multivessel CAD and acceptable } \\
\text { surgical risk, CABG recommended } \\
\text { over PCI } \\
\text { Class IIa (LOE B): Patients with } \\
\text { multivessel CAD and SYNTAX } \leq 22 \text {, } \\
\text { PCI should be considered as alternative } \\
\text { to CABG }\end{array}$ \\
\hline
\end{tabular}

$\overline{A C C F}$, American College of Cardiology Foundation; $A C S$, acute coronary syndrome; $A H A$, American Heart Association; $C A B G$, coronary artery bypass grafting; $C A D$, coronary artery disease; DM, diabetes mellitus; EACTS, European Association for Cardio-Thoracic Surgery; ESC, European Society of Cardiology; LAD, left anterior descending; LIMA, left internal mammary artery; $L M$, left main; $L O E$, level of evidence; $P C I$, percutaneous coronary intervention; STS, The Society of Thoracic Surgeons.

The American guidelines, in general, appear more liberal with the use of PCI in patients with three-vessel disease when low or intermediate complexity is present and more restrictive when LM disease is involved. They are not so closely structured according to the SYNTAX score or other means representing the complexity of the coronary anatomy. Rather, a subset of clinical scenarios is taken into consideration.

Comparisons between American and European guidelines for patients with stable CAD are detailed in Table 2.

Figures 1 and 2 show suggested algorithms, in patients with stable CAD without or with LM coronary artery involvement, to help simplify the decision-making process and to possibly avoid the need for systematic discussion of every patient with locally agreed protocols (adapted from 2013 ESC Guidelines on the management of stable CAD). ${ }^{17}$

\section{BILATERAL INTERNAL MAMMARY ARTERY}

Although nicely presenting the physiological basis for improved graft patency with arterial versus saphenous vein
(SV) bypass conduits, the ACCF/AHA Guidelines are remarkably conservative in their recommendations. Although a large number of studies had already been published supporting the survival benefit of bilateral versus single IMA grafting, the writing committee elected to quote only studies from a single centre in a very subdued recommendation stating that "when anatomically and clinically suitable, use of a second IMA to graft the left circumflex or right coronary artery is reasonable to improve the likelihood of survival and to decrease reintervention rate" (COR IIa, LOE B). Oddly enough, the studies cited to support this recommendation, although carefully performed, reported neither the largest, least selective, nor longest followed patient cohorts available in the literature at the time of guideline generation. ${ }^{18,19}$ Clearly, the absence of prospective randomized control trial (RCT) data may have influenced the task force's recommendations, despite the physiologically sound and increasingly clinically robust evidence supporting bilateral internal mammary artery (BIMA) grafting. The 


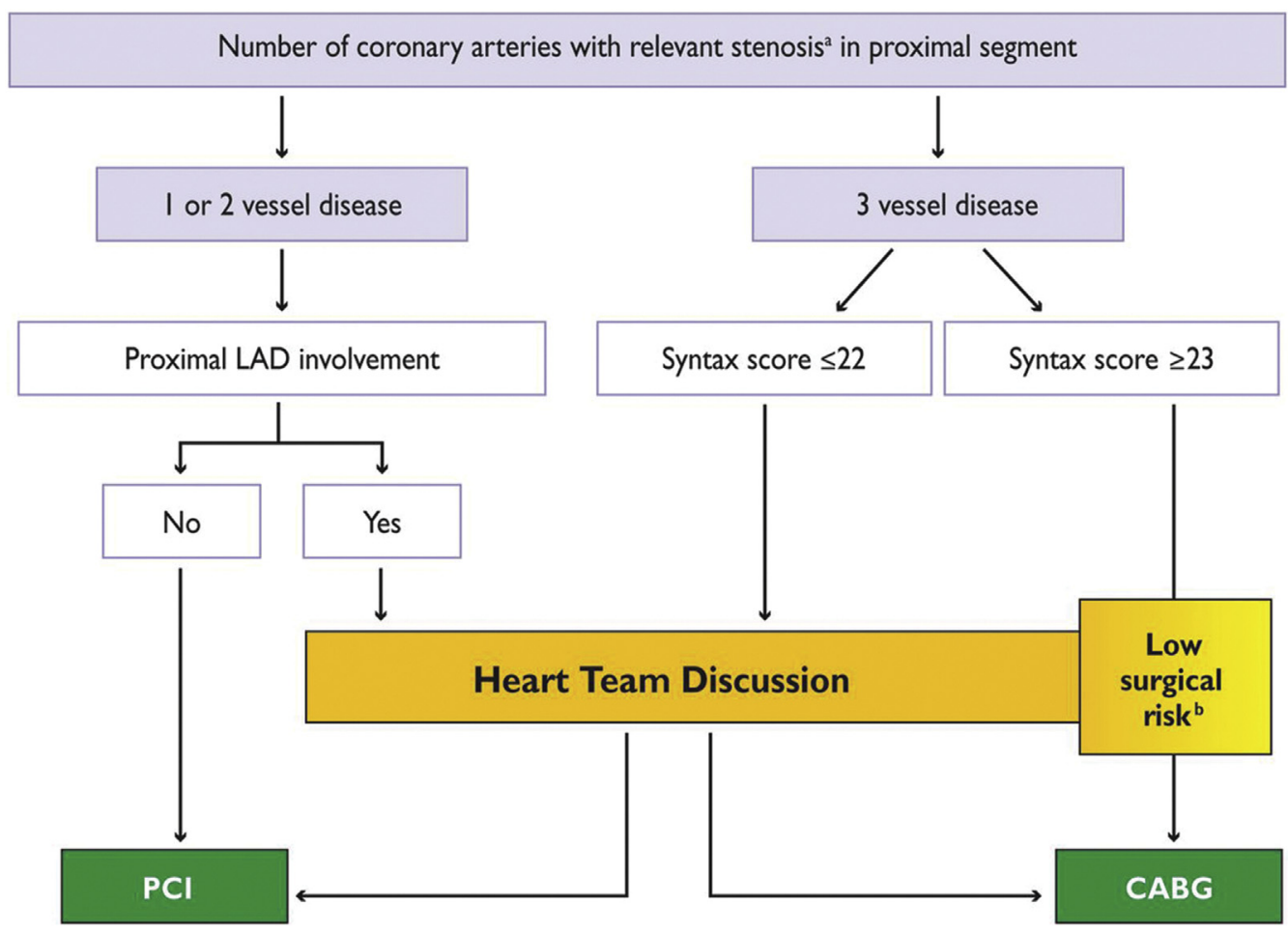

FIGURE 1. Percutaneous coronary intervention or coronary artery bypass graft surgery in stable coronary artery disease without left main coronary artery involvement. ${ }^{\mathrm{a}}>50 \%$ stenosis and proof of ischemia, $>90 \%$ stenosis in two angiographic views or fractional flow reserve $<0.80$. ${ }^{\mathrm{b}} \mathrm{CABG}$ is the preferred option in most patients unless patients' comorbidities or specificities deserve discussion by the Heart Team. According to local practice (time constraints and workload), direct transfer to CABG may be allowed in these low-risk patients, when formal discussion in a multidisciplinary team is not required. $C A B G$, Coronary artery bypass grafting; $L A D$, left anterior descending; $P C I$, percutaneous coronary intervention. (Adapted from ESC/EACTS Guidelines on Myocardial Revascularization 2010; reproduced from reference 17 with permission of Oxford University Press (UK) @ European Society of Cardiology, www.escardio.org/guidelines.)

discussion of the risk of sternal wound infection was not referenced, despite extensive literature on the topic, and no mention was made on the potential impact that the "skeletonized" technique for IMA harvest may have on reducing the risk of sternal infection.

The European guidelines, on the other hand, perhaps due to a large number of supportive studies that have emerged in the years between the release of these two sets of guidelines, ${ }^{18,19}$ are much more supportive of the use of bilateral IMA grafting. The authors specifically address the decreased risk of sternal infection (as well as other potential benefits) with the skeletonized approach to IMA harvest, and directly address the potentially increased risk of mediastinitis, particularly in diabetics and obese patients, with robust reference to the literature. The authors, in the text, conclude that "BIMA grafting is recommended if life expectancy exceeds 5 years and to avoid aortic manipulation." However, in framing the guideline itself, the authors opted for a more conservative tone: "Bilateral IMA grafting should be considered in patients $<70$ years of age," as a COR IIa, LOE B. What is perhaps interesting is that both sets of guidelines classify the recommendation as a COR IIa, LOE B with similar definitions: ACCF/AHA, "Benefit $>>$ Risk, It is reasonable, additional studies needed"; ECS/EACTS, "Weight of evidence in favor, should be considered." Clearly, the data that will emerge from the only prospective RCT on the topic will be eagerly awaited, although, as with any good study, it is likely to raise as many questions as it answers. ${ }^{20}$

\section{RADIAL ARTERY}

This comparison is based on the 2011 ACCF/AHA Guidelines for CABG Surgery, ${ }^{2}$ in concert with the 2014 ESC/EACTS Guidelines on MR. ${ }^{1}$ As a general comment, the European guidelines are more procedurally directive than the American guidelines. The 2014 ACC/AHA/ AATS/PCNA/SCAI/STS focused update ${ }^{4}$ does not contain any new guidelines with respect to radial artery (RA) grafting. The STS has recently published Clinical Practice Guidelines on Arterial Conduits that do address the RA. ${ }^{5}$ The recommendations of the STS Practice Guidelines regarding RA grafting will be outlined as well.

The 2011 ACCF/AHA Guidelines contain a single COR IIb (ie, may be considered), based on LOE B, for the use of the RA for CABG. Specifically, the recommendation 


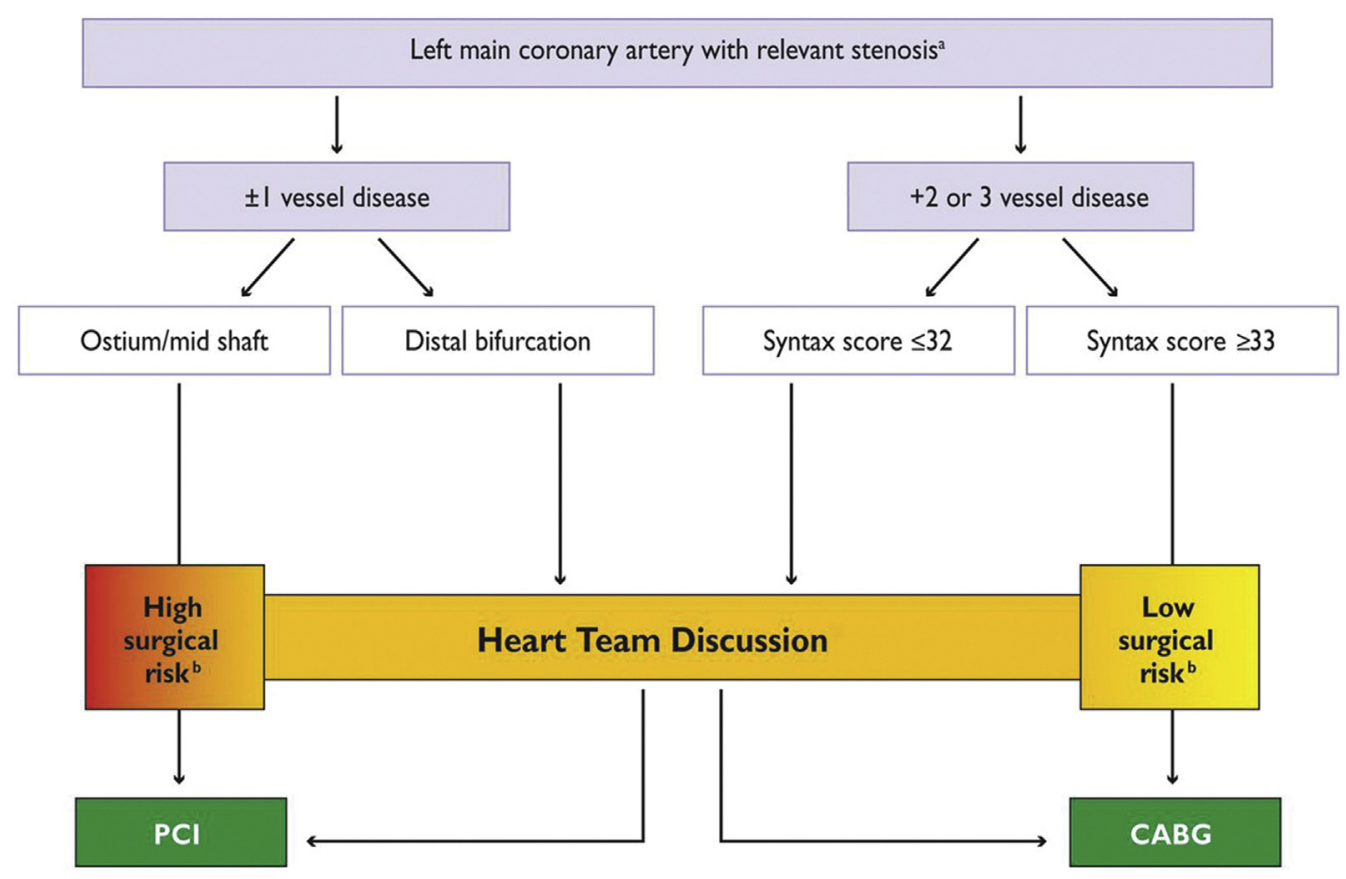

FIGURE 2. Percutaneous coronary intervention or coronary artery bypass graft surgery in stable coronary artery disease with left main coronary artery involvement. ${ }^{\mathrm{a}}>50 \%$ stenosis and proof of ischemia, $>70 \%$ stenosis in two angiographic views or fractional flow reserve $<0.80$. ${ }^{\mathrm{b}}$ Preferred option in general. According to local practice (time constraints and workload), direct decision may be taken without formal multidisciplinary discussion, but preferably with locally agreed protocols. $C A B G$, Coronary artery bypass grafting; $P C I$, percutaneous coronary intervention. (Adapted from ESC/EACTS Guidelines on Myocardial Revascularization 2010; reproduced from reference 17 with permission of Oxford University Press (UK) @ European Society of Cardiology, www.escardio.org/guidelines.)

emphasizes the importance of a severe proximal stenosis when using an RA, which was defined as left-sided stenosis of $>70$ or $>90 \%$ (ie, critical) for a right-sided target vessel. The text is short, but emphasizes that RAs are prone to spasm and/or atrophy when directed to a moderately rather than a severely narrowed coronary artery. It is well recognized that arterial grafts including the RA may fail not only by occlusion but can also remain patent while becoming extremely narrowed and non-functional (string sign). ${ }^{21}$ Evidence exists that the RA is probably more dependent on the severity of the proximal target vessel stenosis than the IMA. ${ }^{22,23}$ The 2011 ACCF/AHA Guidelines also include a further COR III, LOE C, regarding the importance of not grafting the right coronary artery with an arterial graft (RA not specifically mentioned), unless it has a critical stenosis.

The 2014 ESC/EACTS Guidelines were written following the publication of several additional important randomized and observational studies concerning RA grafting. ${ }^{24-26}$ One table in these guidelines summarizes the early, mid and late patency of the RA in addition to SV grafts and left and right IMAs - RA patency exceeds that of SVs but is likely less than the left or right IMA. ${ }^{1}$ Also, late survival and event-free survival are enhanced. Consequently, these recent guidelines more strongly recommend the RA (COR I, LOE $\mathrm{B})$ than the American guidelines, but again, like the American guidelines, emphasize the importance of a high-grade stenosis of the native coronary artery. The guidelines do not stipulate the severity; however, in the text, the authors describe that patency of the RA is strongly affected for lesions $<70 \%$. In the text, the writers conclude that the RA is a reasonable alternative for a second arterial graft when BIMA harvesting is contraindicated due to increased risks of sternal infection.

The very recent STS Practice Guidelines ${ }^{5}$ recommend a Heart Team approach for decisions regarding revascularization, including the type of grafts (COR I, LOE C). The STS Practice Guidelines recommend a second arterial graft, either a second IMA or an RA (COR IIa, LOE B in appropriate patients), recognizing that patient comorbidities affect the risks and relative benefits of different approaches. Again, the RA should be directed to a coronary artery with a severe stenosis (COR IIa, LOE B); in addition, pharmacological dilatation is recommended intraoperatively and perioperatively (COR IIa, LOE B), but there is insufficient evidence to warrant any recommendation regarding longer-term use of pharmacological dilatation.

The American and European scientific societies recognize the prognostic importance of the severity of target vessel stenosis for radial graft patency based on visual assessment by coronary angiogram. Physiologically rather than anatomically defined stenosis as assessed by fractional flow reserve (FFR) has prognostic importance in 
percutaneous revascularization, and both the American and European guidelines recommend this practice for PCI. ${ }^{27-32}$ There is some evidence that FFR-guided coronary surgery is associated with improved graft patency. ${ }^{33,34}$ Whether FFR, or FFR in association with anatomical severity will supplant visual assessment for radial grafting specifically, or coronary surgery in general, is unclear at this stage.

\section{TOTAL ARTERIAL REVASCULARIZATION}

In a somewhat uncharacteristic fashion, the issue of total arterial revascularization (TAR) is mentioned in the ACCF/ AHA Guidelines as a COR IIb ( $\mathrm{LOE} \mathrm{C)} \mathrm{-} \mathrm{"} \mathrm{may} \mathrm{be} \mathrm{reason-}$ able in patients $\leq 60$ years of age with few or no comorbidities"-without further discussion or reference to any specific literature. A similar lack of direct discussion of the topic is apparent in the European guidelines, although two recommendations are made: One supporting TAR for patients with poor vein quality (COR I, LOE C, without reference, presumably based on logical and prior recommendations for complete revascularization), and the other advocating that TAR should be considered in patients with reasonable life expectancy (COR IIA, LOE B) based on a single reference. ${ }^{35}$ Although thorough in its practical exploration of potential conduits, only two studies were cited in this article, which directly addressed the issue of TAR. ${ }^{36,37}$ Perhaps, the reticence of the guideline writing committees reflects the relative paucity of data on the topic. Although considerable clinical data support the use of multiple arterial grafting, TAR only comprises a small portion of such reports. ${ }^{36-39}$ Comparisons are usually with single IMA grafting strategies, and differential use of IMAs and RAs, as well as the issue of in situ versus free $\mathrm{Y}$ and $\mathrm{T}$ grafting, make comparisons difficult. Concerns regarding the use of RAs, which have been used for interventional procedures, further complicate the issue. ${ }^{40}$ The major question emerging is whether or not a strategy of TAR provides incremental benefit compared with BIMA or even single IMA/RA grafting. Reports to date have been encouraging but inconsistent. ${ }^{4-43}$ Clearly, more compelling data will be needed to warrant guideline-based changes in practice.

\section{ON- VERSUS OFF-PUMP}

Although the ACC, AHA and associated societies have performed a recent focused update for the diagnosis and management of patients with stable ischemic heart disease, ${ }^{4}$ this did not address the issues of off-pump and on-pump CABG, and optimal conduit selection. Therefore, the comparisons and comments on these topics are solely based on the 2011 ACCF/AHA Guidelines for CABG Surgery ${ }^{2}$ and the 2014 ESC/EACTS Guidelines on MR. ${ }^{1}$

The ACCF/AHA Guidelines directly address the use of cardiopulmonary bypass (CPB) as well as the more specific issue of off-pump versus on-pump CABG. Interestingly however, despite a somewhat scholarly review of the physiological basis for the Systemic Inflammatory Response Syndrome (SIRS) and its potential clinical sequelae, especially as regards neurocognitive and renal dysfunction, and a focused review of the available evidence comparing onand off-pump approaches, the writing committee elected to offer no guideline recommendations regarding either issue. Multiple strategies for mitigating potential mediators of surgical morbidity, most specifically as regards renal and neurological dysfunction-cell-saver processing of shed blood, modulating neutrophil activation, steroid and immunoglobulin $\mathrm{G}$ administration, use of coated and/or mini-CPB circuits-were all mentioned without convincing arguments supporting their efficacy. It is perhaps this absence of compelling evidence that has prompted the European colleagues to avoid directly addressing the topic.

Regarding the issue of off- versus on-pump approaches to CABG surgery, the ACC/AHA writing committee was clearly concerned by the apparent disparities in the data. The equipoise of early small, prospective RCTs ${ }^{44,45}$ and the negative finding of the somewhat controversial ROOBY trial ${ }^{46}$ have left the authors with an exploration of registry data which was equally inconclusive, even in the high-risk patient population. ${ }^{47-49}$ The concluding remarks focused on the avoidance of aortic manipulation, regardless of an on- versus off-pump approach in patients with evidence of aortic atherosclerotic disease, acknowledging that this may be more readily achieved with an off-pump approach. It was, however, noted that patients with unstable hemodynamics may be more readily managed with an on-pump approach. In short, although there may be patients for whom one approach or the other may be preferable, given adequate surgical expertise, "most surgeons consider either approach to be reasonable for the majority of subjects undergoing CABG," no formal recommendation was given.

The European Task Force, on the other hand, had the benefit of more robust data. Two additional prospective RCTs, focusing on elderly and high-risk patients, had failed to demonstrate a difference in 30-day or 1-year outcomes when on- or off-pump CABG was performed by experienced teams. ${ }^{50,51}$ Interestingly, based on some of the same data available to the American group, ${ }^{47,52}$ the Europeans were more convinced of the potential value of off-pump surgery in high-risk patients, especially with regard to stroke, and recommended that off-pump CABG be "considered" for subgroups of high-risk patients in high-volume offpump centres as a COR IIa based on LOE B. Like their American colleagues, the European group was even more definitive regarding minimization of aortic manipulation and "recommended" off-pump CABG and/or no touch on-pump techniques for patients with significant atherosclerotic aortic disease as a COR I with LOE B.

Subsequent studies have suggested that complexity of the issue may revolve around surgical expertise and patient 
selection, in that the short-term potential benefits for offpump need to be balanced against the long-term apparent benefits for the on-pump approach. The reduced graft patency, demonstrated in a meta-analysis of RCTs ${ }^{53}$ seems consistent with data emerging from a meta-analysis of RCTs and observational studies in over 100000 patients, which demonstrated a worse 5-year survival among offas opposed to on-pump patients. ${ }^{54}$ Interestingly, current evidence suggests that the difference in graft patency may be limited to SV grafts, suggesting a potential role for increased use of arterial conduits regardless of the CPB strategy employed. Hopefully, by the time the current guidelines are updated, better evidence will be available to identify, in equally experienced hands, which patients are mostly likely to benefit from which approach.

\section{MINIMALLY INVASIVE DIRECT CORONARY ARTERY BYPASS AND HYBRID REVASCULARIZATION}

Neither the 2011 American guidelines $^{2}$ nor the subsequent update ${ }^{4}$ included any recommendations regarding minimally invasive surgery. The 2014 European guidelines ${ }^{1}$ did advise that minimally invasive direct coronary artery bypass (MIDCAB) be considered for isolated LAD disease (COR IIa, LOE C). In the text, the writers concede that while the safety and efficacy of MIDCAB are similar to that achieved with conventional on- or off-pump coronary surgery, these approaches do achieve benefits in terms of shorter length of hospital stay and a better quality of life early following surgery. The text in the American guidelines acknowledges the potential benefits of a minimally invasive approach, namely avoidance of sternal complications, earlier recovery and enhanced cosmesis, and also mentions robotic-assisted, endoscopic approaches as the most minimally invasive technique. ${ }^{55}$ However, the 2011 American guidelines focus mainly on the limitations of a small anterolateral thoracic incision (or other incisions short of a full sternotomy), in terms of lack of exposure of all coronary territories and the aorta.

Efforts to provide complete revascularization with minimal surgical incisions have led to interest in hybrid revascularization - namely isolated LAD bypass with an IMA graft and stenting of the right coronary artery and/or circumflex artery territories, either simultaneously, or as a staged procedure. Hybrid revascularization acknowledges the relative benefit of an IMA for LAD revascularization, and the reduced invasiveness of PCI for non-LAD targets, in concert with the progressively improved results of PCI, particularly with later-generation drug-eluting stents. Although hybrid revascularization is commonly understood to involve a minimally invasive approach for left internal mammary artery (LIMA)-LAD bypass (MIDCAB, robotic), a full sternotomy for LIMA-LAD bypass in conjunction with PCI is compatible with the definition, ${ }^{56}$ and likely to be utilized by surgeons more often than one of the minimally invasive methods. ${ }^{57}$

Consequently, the American guidelines do include specific recommendations on hybrid revascularization. The American guidelines recommend hybrid revascularization as COR IIa, LOE B in multivessel disease patients when conventional surgical revascularization or PCI is anticipated to be more challenging or hazardous, ${ }^{58}$ for surgical patients due to a hostile aorta and/or poor targets and/or limited conduit, and for PCI patients when percutaneous revascularization of the $\mathrm{LAD}$ is expected to be problematic due to local angiographic features. The American guidelines do make a further COR IIb, LOE C as an alternative to $\mathrm{CABG}$ or $\mathrm{PCI}$ - this approach is intended to minimize risk and improve the benefits relative to isolated PCI or CABG. Presumably, a Heart Team would help make such a decision although the writers did not further elaborate on the context of this recommendation. Whereas the European guidelines are generally more directive about procedural aspects of revascularization, they only suggest that hybrid approaches may be considered in specific patient cohorts and in experienced institutions (COR IIb, LOE C). In the text, it becomes clearer that the authors have adopted a broader interpretation of hybrid revascularization by including not only patients treated with coronary surgery and PCI, but also PCI and other cardiac operations. The European writing committee did make a strong recommendation to avoid aortic manipulation in patients with a very hostile ascending aorta, COR I, LOE B. The data supporting or refuting the MIDCAB and hybrid revascularization approaches are substantially more limited than for other procedural aspects of coronary bypass surgery. ${ }^{57}$

\section{DIABETIC PATIENTS}

The importance of diabetes mellitus in the selection of revascularization strategy was recognized in the 2014 ACC/AHA/AATS/PCNA/SCAI/STS focused update ${ }^{4}$ and the 2014 ESC/EACTS Guidelines on MR $^{1}$ (Table 2). While both sets of guidelines consider CABG to be a COR I in diabetics, the European authors provide a COR IIa for PCI in diabetics with low SYNTAX scores. Beyond the recommendation for use of the LIMA to LAD, the American guidelines do not delve into the issue of how diabetes might influence surgical grafting strategy. The European task force directly discusses the potential impact of BIMA grafting on the diabetic patient and suggests that BIMA grafting should be considered (COR IIa, LOE B). Although both sets of guidelines express concern regarding the potential for increased risk of sternal wound infection with IMA harvest in diabetic patients, it was not until the recent release of the STS Clinical Practice Guidelines on Arterial Conduits for $\mathrm{CABG}^{5}$ that specific strategies for reducing this risk through the use of a "skeletonized" harvest approach is directly addressed as a COR IIa, LOE B. 
Although diabetes is an established risk variable for infection, the risk of radial harvest site infection is low, with or without diabetes. Diabetes was associated with an increased risk of graft occlusion at 1 year and 7.5 years postoperatively in the RAPS studies ${ }^{59,60}$; however, the use of an RA compared with SV grafting was associated with reduced graft occlusion in the diabetic cohort. In observational studies, the use of an RA as a second arterial conduit proved to be protective in terms of late survival. ${ }^{61,62}$

\section{GUIDELINES AND TREATMENT REALITY}

Surgeons in numerous countries have complained about an uncontrolled overuse of PCI over CABG with everincreasing rates of PCI use and falling numbers of isolated CABG procedures, particularly for stable ischemic heart disease. In this regard, the report of the OECD (Organization for Economy Cooperation and Development) gives more specific information on the use of revascularization techniques in the different European countries, the USA and some other selected nations. An average rate of 218 coronary revascularization procedures per 100000 population is reported with an average PCI proportion of $72 \%$ performed in 2013. ${ }^{63}$ Across countries, there is a tremendous variation in these figures, with the highest revascularization rate of about 435 procedures per 100000 in Germany, triggered by the enormous rate of PCI (roughly 360 per $100,000)$ resulting in a PCI proportion of $84 \%$. A closer look at the nationally published annual data ${ }^{64,65}$ suggests an even greater disparity in the rates of PCI and CABG. For example, in Germany, an absolute number of 40000 isolated CABGs and 360000 PCIs were reported in 2014, yielding a PCI/CABG ratio of 9:1.

In the USA, Appropriate Use Criteria for coronary revascularization were published in $2009 .^{66}$ The overall rates of PCI have declined in the USA subsequent to the publication of the Appropriate Use Criteria ${ }^{67,68}$ - this drop is entirely related to a substantial decrease in the rates of PCI for stable or non-acute ischemic heart disease, while the rates for acute indication have remained stable. Furthermore, the proportion of PCI procedures judged to be inappropriate has declined as well. ${ }^{68}$

The fundamental differences in the worldwide use of PCI and CABG may be caused by different healthcare system structures with limitations in capacity and access to treatment options in the health service or may be driven by reimbursement forces and financial aspects. To what extent and under which conditions revascularization procedures are performed under adherence to the relevant European and American CAD guidelines cannot be substantially evaluated on the basis of most national data sources. Necessary items like the treatment of threevessel disease and/or LM stenosis, as well as distinction between stable CAD and acute MI, are not systematically monitored in parallel to the guidelines although medical quality assessment systems are established in the majority of the mentioned countries.

The use of a Heart Team approach — strongly indicated in the guidelines from both sides of the Atlantic- should partially address the concern regarding the overuse of PCI versus CABG. However, the extent to which a formal Heart Team involvement or institutional protocols are applied, as recommended in the guidelines, remains unclear. To improve guideline implementation and prevent malpractice, it is of major importance in the future to apply mandatory, more specific data sets in quality assessment and allow for the required transparency and control. Even more formal measures comparable to structured tumor boards in oncology and reimbursement of treatment depending on guideline adherence should be put under consideration.

\section{CONCLUSIONS}

With this editorial, we could convincingly outline a broadly coherent similarity between the 2011 ACCF/AHA Guidelines for CABG Surgery, including supplementary updates over the recent years, and the 2014 ESC/EACTS Guidelines on MR. Whenever the same patient cohorts were mentioned, the American and European guidelines present astonishingly similar major recommendation in at least 6 occasions. Another 11 major recommendations are slightly different, albeit pointing clearly in the same direction. We identified only two instances in which the guidelines present meaningfully different recommendations, which most certainly were related to the time gap in between the creation of the two sets of guidelines.

Particularly for areas of highest controversy between surgeons and cardiologists, the recommendations for revascularization of three-vessel disease and LM disease in stable coronary heart disease, a high level of concordance between both guidelines was demonstrated, even though the timing of guidelines release differed in the basic versions by 3 years. Thus, the ESC/EACTS Guidelines are, in fact, more contemporary and relevant as they incorporate the 5-year results of the SYNTAX trial and more recent metaanalyses on studies for multivessel revascularization and LM procedures. Besides very specific recommendations including the complexity of the coronary findings, there is general consensus in recommending bypass surgery either as an equivalent alternative or preferential procedure compared with PCI, whenever a significant proximal LAD stenosis is present.

The practical and scientific value of both guidelines is undoubtedly proved by a worldwide endorsement through the specific cardiological and cardio-surgical medical societies, even outside the USA and Europe, often with additional independent national guidelines adopting these recommendations. However, it has also become evident that there is a significant numeric imbalance in total rates and proportion of the applied techniques by official healthcare figures; 
allegations of PCI overuse have frequently been suggested by surgeons, non-invasive cardiologists and general practitioners, on the one hand, or even by health insurance companies in the interest of patient care, on the other.

To achieve medically reasonable diagnostics and treatments as proposed by medical guidelines along with cost-effective distribution of healthcare resources in the cardiovascular health system, it is mandatory to achieve transparency and control under conditions of systematic and reliable data monitoring and reporting systems in parallel to the major decision-making criteria being used for stratification according to the treatment algorithms. For practical use, the application of either institutional protocols or individual case-by-case Heart Team decisions is strongly dependent on the confidence and quality of collaboration between the interventional and non-interventional cardiologists and the cardiac surgeons. Especially in institutions with obvious disagreement or malfunction, a regulatory role of the hospital administration or even of state healthcare organizations may be necessary to ensure correct guideline implementation in the interest of evidence-based medicine.

The ultimate goal of guidelines is to create a framework to facilitate patient-focused care. To this end, US and European cardiovascular specialty societies strongly recommend a Heart Team approach (COR I). Marked regional variation in the rates of different medical interventions, such as the ratio of PCI to coronary bypass operations, is usually unrelated to patient differences but rather differences in health practitioner behavior or healthcare systems. A marked change in the pattern of practice of PCI for stable ischemic heart disease in the USA followed the publication of the Appropriate Use Criteria, showing that physician behavior can change with appropriate incentives. Creating systems with appropriate inducements at the hospital or regional/national level, or along with private insurers where appropriate, should ensure that adherence is high.

There is a sense in the global cardiac surgical community that revascularization decision-making is not evidencebased. Heart Teams can initiate patient discussions using the treatment algorithms as outlined in the revascularization guidelines-however as doctors, clinical decision-making typically requires a more comprehensive understanding of the unique characteristics of the individual patient. For patient-focused care, each specialty needs to hear the other colleague's viewpoint. When this fails to happen, we need to remain cognizant of the fact that it is the patient who ultimately loses from dysfunctional interactions - market share is not the issue. And remember that cardiologists and cardiac surgeons are on the same team-the Heart Team.

\section{References}

1. Kolh P, Windecker S, Alfonso F, Collet JP, Cremer J, Falk V, et al. 2014 ESC/ EACTS guidelines on myocardial revascularization: the task force on myocardial revascularization of the European Society of Cardiology (ESC) and the European
Association for Cardio-Thoracic Surgery (EACTS). Developed with the special contribution of the European Association of Percutaneous Cardiovascular Interventions (EAPCI). Eur J Cardiothorac Surg. 2014;46:517-92.

2. Hillis LD, Smith PK, Anderson JL, Bittl JA, Bridges CR, Byrne JG, et al. 2011 ACCF/AHA guideline for coronary artery bypass graft surgery. A report of the American College of Cardiology Foundation/American Heart Association Task Force on Practice Guidelines. Developed in collaboration with the American Association for Thoracic Surgery, Society of Cardiovascular Anesthesiologists, and Society of Thoracic Surgeons. J Am Coll Cardiol. 2011:58:e123-210.

3. Fihn SD, Gardin JM, Abrams J, Berra K, Blankenship JC, Dallas AP, et al. 2012 ACCF/AHA/ACP/AATS/PCNA/SCAI/STS guideline for the diagnosis and management of patients with stable ischemic heart disease: a report of the American College of Cardiology Foundation/American Heart Association Task Force on Practice Guidelines, and the American College of Physicians, American Association for Thoracic Surgery, Preventive Cardiovascular Nurses Association, Society for Cardiovascular Angiography and Interventions, and Society of Thoracic Surgeons. J Am Coll Cardiol. 2012;60:e44-164.

4. Fihn SD, Blankenship JC, Alexander KP, Bittl JA, Byrne JG, Fletcher BJ, et al. 2014 ACC/AHA/AATS/PCNA/SCAI/STS focused update of the guideline for the diagnosis and management of patients with stable ischemic heart disease: a report of the American College of Cardiology/American Heart Association Task Force on Practice Guidelines, and the American Association for Thoracic Surgery, Preventive Cardiovascular Nurses Association, Society for Cardiovascular Angiography and Interventions, and Society of Thoracic Surgeons. J Thorac Cardiovasc Surg. 2015;149:e5-23.

5. Aldea GS, Bakaeen FG, Pal J, Fremes S, Head SJ, Sabik J, et al. The Society of Thoracic Surgeons Clinical Practice Guidelines on Arterial Conduits for coronary artery bypass grafting. Ann Thorac Surg. 2016;101:801-9.

6. Feit F, Brooks MM, Sopko G, Keller NM, Rosen A, Krone R, et al. Long-term clinical outcome in the Bypass Angioplasty Revascularization Investigation Registry: comparison with the randomized trial. BARI Investigators. Circulation. 2000; 101:2795-802.

7. King SB III, Barnhart HX, Kosinski AS, Weintraub WS, Lembo NJ, Petersen JY, et al. Angioplasty or surgery for multivessel coronary artery disease: comparison of eligible registry and randomized patients in the EAST trial and influence of treatment selection on outcomes. Emory Angioplasty versus Surgery Trial Investigators. Am J Cardiol. 1997;79:1453-9.

8. Bonzel T, Schachinger V, Dorge H. Description of a Heart Team approach to coronary revascularization and its beneficial long-term effect on clinical events after PCI. Clin Res Cardiol. 2015 [Epub ahead of print].

9. Yates MT, Soppa GK, Valencia O, Jones S, Firoozi S, Jahangiri M. Impact of European Society of Cardiology and European Association for Cardiothoracic Surgery Guidelines on myocardial revascularization on the activity of percutaneous coronary intervention and coronary artery bypass graft surgery for stable coronary artery disease. J Thorac Cardiovasc Surg. 2014;147:606-10.

10. Long J, Luckraz H, Thekkudan J, Maher A, Norell M. Heart team discussion in managing patients with coronary artery disease: outcome and reproducibility. Interact CardioVasc Thorac Surg. 2012;14:594-8.

11. O'Gara PT, Kushner FG, Ascheim DD, Casey DE Jr, Chung MK, de Lemos JA, et al. 2013 ACCF/AHA guideline for the management of ST-elevation myocardial infarction: a report of the American College of Cardiology Foundation/ American Heart Association Task Force on Practice Guidelines. Circulation. 2013;127:e362-425.

12. Amsterdam EA, Wenger NK, Brindis RG, Casey DE Jr, Ganiats TG, Holmes DR Jr, et al. 2014 AHA/ACC guideline for the management of patients with non-st-elevation acute coronary syndromes: a report of the American College of Cardiology/American Heart Association Task Force on Practice Guidelines. J Am Coll Cardiol. 2014;64:e139-228.

13. Hamm CW, Bassand JP, Agewall S, Bax J, Boersma E, Bueno H, et al. ESC guidelines for the management of acute coronary syndromes in patients presenting without persistent ST-segment elevation: the task force for the management of acute coronary syndromes (ACS) in patients presenting without persistent STsegment elevation of the European Society of Cardiology (ESC). Eur Heart J. 2011;32:2999-3054.

14. Southern DA, Izadnegahdar M, Humphries KH, Gao M, Wang F, Knudtson ML, et al. Trends in wait times for cardiac revascularization. Can J Cardiol. 2011;27: 262-7.

15. Sianos G, Morel MA, Kappetein AP, Morice MC, Colombo A, Dawkins K, et al. The SYNTAX score: an angiographic tool grading the complexity of coronary artery disease. EuroIntervention. 2005;1:219-27. 
16. Mohr FW, Morice MC, Kappetein AP, Feldman TE, Stahle E, Colombo A, et al. Coronary artery bypass graft surgery versus percutaneous coronary intervention in patients with three-vessel disease and left main coronary disease: 5year follow-up of the randomised, clinical SYNTAX trial. Lancet. 2013;381: 629-38.

17. Montalescot G, Sechtem U, Achenbach S, Andreotti F, Arden C, Budaj A, et al. 2013 ESC guidelines on the management of stable coronary artery disease: the task force on the management of stable coronary artery disease of the European Society of Cardiology. Eur Heart J. 2013;34:2949-3003.

18. Yi G, Shine B, Rehman SM, Altman DG, Taggart DP. Effect of bilateral internal mammary artery grafts on long-term survival: a meta-analysis approach. Circulation. 2014; 130:539-45.

19. Takagi H, Goto SN, Watanabe T, Mizuno Y, Kawai N, Umemoto T. A meta-analysis of adjusted hazard ratios from 20 observational studies of bilateral versus single internal thoracic artery coronary artery bypass grafting. J Thorac Cardiovasc Surg. 2014;148:1282-90.

20. Taggart DP, Altman DG, Gray AM, Lees B, Nugara F, Yu LM, et al. Randomized trial to compare bilateral vs. single internal mammary coronary artery bypass grafting: 1-year results of the Arterial Revascularisation Trial (ART). Eur Heart J. 2010;31:2470-81

21. Miwa S, Desai N, Koyama T, Chan E, Cohen EA, Fremes SE. Radial artery angiographic string sign: clinical consequences and the role of pharmacologic therapy. Ann Thorac Surg. 2006;81:112-8.

22. Desai ND, Naylor CD, Kiss A, Cohen EA, Feder-Elituv R, Miwa S, et al. Impact of patient and target-vessel characteristics on arterial and venous bypass graft patency: insight from a randomized trial. Circulation. 2007;115:684-91.

23. Tatoulis J, Buxton BF, Fuller JA. The right internal thoracic artery: the forgotten conduit-5,766 patients and 991 angiograms. Ann Thorac Surg. 2011;92:9-15.

24. Deb S, Cohen EA, Singh SK, Une D, Laupacis A, Fremes SE. Radial artery and saphenous vein patency more than 5 years after coronary artery bypass surgery: results from RAPS (Radial Artery Patency Study). J Am Coll Cardiol. 2012;60: 28-35.

25. Achouh P, Isselmou KO, Boutekadjirt R, D’Alessandro C, Pagny JY, Fouquet R, et al. Reappraisal of a 20-year experience with the radial artery as a conduit for coronary bypass grafting. Eur J Cardiothorac Surg. 2012;41:87-92.

26. Tranbaugh RF, Dimitrova KR, Friedmann P, Geller CM, Harris LJ, Stelzer P, et al. Coronary artery bypass grafting using the radial artery: clinical outcomes, patency, and need for reintervention. Circulation. 2012;126:S170-5.

27. De Bruyne B, Fearon WF, Pijls NH, Barbato E, Tonino P, Piroth Z, et al. Fractional flow reserve-guided PCI for stable coronary artery disease. $N$ Engl J Med. 2014;371:1208-17.

28. De Bruyne B, Pijls NH, Kalesan B, Barbato E, Tonino PA, Piroth Z, et al. Fractional flow reserve-guided PCI versus medical therapy in stable coronary disease. N Engl J Med. 2012;367:991-1001.

29. Pijls NH, Fearon WF, Tonino PA, Siebert U, Ikeno F, Bornschein B, et al. Fractional flow reserve versus angiography for guiding percutaneous coronary intervention in patients with multivessel coronary artery disease: 2-year follow-up of the FAME (Fractional Flow Reserve Versus Angiography for Multivessel Evaluation) study. J Am Coll Cardiol. 2010;56:177-84.

30. Tonino PA, De Bruyne B, Pijls NH, Siebert U, Ikeno F, Veer M, et al. Fractional flow reserve versus angiography for guiding percutaneous coronary intervention. N Engl J Med. 2009;360:213-24.

31. Nascimento BR, Belfort AF, Macedo FA, Sant'Anna FM, Pereira GT, Costa MA, et al. Meta-analysis of deferral versus performance of coronary intervention based on coronary pressure-derived fractional flow reserve. Am J Cardiol. 2015;115:385-91.

32. Zhang D, Lv S, Song X, Yuan F, Xu F, Zhang M, et al. Fractional flow reserve versus angiography for guiding percutaneous coronary intervention: a meta-analysis. Heart. 2015;101:455-62

33. Toth G, De Bruyne B, Casselman F, De Vroey F, Pyxaras S, Di Serafino L, et al. Fractional flow reserve-guided versus angiography-guided coronary artery bypass graft surgery. Circulation. 2013;128:1405-11.

34. Botman CJ, Schonberger J, Koolen S, Penn O, Botman H, Dib N, et al. Does stenosis severity of native vessels influence bypass graft patency? A prospective fractional flow reserve-guided study. Ann Thorac Surg. 2007;83:2093-7.

35. Tatoulis J. Total arterial coronary revascularization-patient selection, stenoses, conduits, targets. Ann Cardiothorac Surg. 2013;2:499-506.

36. Zacharias A, Schwann TA, Riordan CJ, Durham SJ, Shah AS, Engoren M, et al. Late outcomes after radial artery versus saphenous vein grafting during reoperative coronary artery bypass surgery. J Thorac Cardiovasc Surg. 2010;139: 1511-8.
37. Muneretto C, Bisleri G, Negri A, Manfredi J, Metra M, Nodari S, et al. Total arterial myocardial revascularization with composite grafts improves results of coronary surgery in elderly: a prospective randomized comparison with conventional coronary artery bypass surgery. Circulation. 2003;108(Suppl 1):II29-33.

38. Buxton BF, Shi WY, Tatoulis J, Fuller JA, Rosalion A, Hayward PA. Total arterial revascularization with internal thoracic and radial artery grafts in triple-vessel coronary artery disease is associated with improved survival. J Thorac Cardiovasc Surg. 2014;148:1238-43.

39. Shi WY, Hayward PA, Tatoulis J, Rosalion A, Newcomb AE, Fuller JA, et al. Are all forms of total arterial revascularization equal? A comparison of single versus bilateral internal thoracic artery grafting strategies. J Thorac Cardiovasc Surg. 2015;150:1526-34.

40. Kamiya H, Ushijima T, Kanamori T, Ikeda C, Nakagaki C, Ueyama K, et al. Use of the radial artery graft after transradial catheterization: is it suitable as a bypass conduit? Ann Thorac Surg. 2003;76:1505-9.

41. Grau JB, Kuschner CE, Johnson CK, Ferrari G, Zapolanski A, Brizzio ME, et al. The effects of using a radial artery in patients already receiving bilateral internal mammary arteries during coronary bypass grafting: 30-day outcomes and 14-year survival in a propensity-matched cohort. Eur J Cardiothorac Surg. 2016;49:203-10.

42. Glineur D. Importance of the third arterial graft in multiple arterial grafting strategies. Ann Cardiothorac Surg. 2013;2:475-80.

43. Guru V, Fremes SE, Tu JV. How many arterial grafts are enough? A populationbased study of midterm outcomes. J Thorac Cardiovasc Surg. 2006;131: 1021-8.

44. Nathoe HM, van Dijk D, Jansen EW, Suyker WJ, Diephuis JC, van Boven WJ et al. A comparison of on-pump and off-pump coronary bypass surgery in lowrisk patients. $N$ Engl J Med. 2003;348:394-402.

45. Khan NE, De Souza A, Mister R, Flather M, Clague J, Davies S, et al A randomized comparison of off-pump and on-pump multivessel coronaryartery bypass surgery. $N$ Engl J Med. 2004;350:21-8.

46. Shroyer AL, Grover FL, Hattler B, Collins JF, McDonald GO, Kozora E, et al On-pump versus off-pump coronary-artery bypass surgery. $N$ Engl $\mathrm{J} \mathrm{Med}$. 2009;361:1827-37.

47. Puskas JD, Thourani VH, Kilgo P, Cooper W, Vassiliades T, Vega JD, et al. Offpump coronary artery bypass disproportionately benefits high-risk patients. Ann Thorac Surg. 2009;88:1142-7.

48. Moller CH, Perko MJ, Lund JT, Andersen LW, Kelbaek H, Madsen JK, et al. No major differences in 30-day outcomes in high-risk patients randomized to offpump versus on-pump coronary bypass surgery: the best bypass surgery trial. Cir culation. 2010;121:498-504.

49. Hannan EL, Wu C, Smith CR, Higgins RS, Carlson RE, Culliford AT, et al Off-pump versus on-pump coronary artery bypass graft surgery: differences in short-term outcomes and in long-term mortality and need for subsequent revascularization. Circulation. 2007;116:1145-52.

50. Lamy A, Devereaux PJ, Prabhakaran D, Taggart DP, Hu S, Paolasso E, et al. Effects of off-pump and on-pump coronary-artery bypass grafting at 1 year. $N$ Engl J Med. 2013;368:1179-88.

51. Diegeler A, Borgermann J, Kappert U, Breuer M, Boning A, Ursulescu A, et al. Off-pump versus on-pump coronary-artery bypass grafting in elderly patients. N Engl J Med. 2013;368:1189-98.

52. Sedrakyan A, Wu AW, Parashar A, Bass EB, Treasure T. Off-pump surgery is associated with reduced occurrence of stroke and other morbidity as compared with traditional coronary artery bypass grafting: a meta-analysis of systematically reviewed trials. Stroke. 2006;37:2759-69.

53. Zhang B, Zhou J, Li H, Liu Z, Chen A, Zhao Q. Comparison of graft patency between off-pump and on-pump coronary artery bypass grafting: an updated metaanalysis. Ann Thorac Surg. 2014;97:1335-41.

54. Takagi H, Umemoto T. Worse long-term survival after off-pump than on-pump coronary artery bypass grafting. J Thorac Cardiovasc Surg. 2014;148:1820-9.

55. Bonatti J, Lee JD, Bonaros N, Schachner T, Lehr EJ. Robotic totally endoscopic multivessel coronary artery bypass grafting: procedure development, challenges, results. Innovations. 2012;7:3-8.

56. Harskamp RE, Bonatti JO, Zhao DX, Puskas JD, de Winter RJ, Alexander JH, et al. Standardizing definitions for hybrid coronary revascularization. J Thorac Cardiovasc Surg. 2014;147:556-60.

57. Harskamp RE, Brennan JM, Xian Y, Halkos ME, Puskas JD, Thourani VH, et al Practice patterns and clinical outcomes after hybrid coronary revascularization in the United States: an analysis from the Society of Thoracic Surgeons adult cardiac database. Circulation. 2014;130:872-9.

58. Panoulas VF, Colombo A, Margonato A, Maisano F. Hybrid coronary revascularization: promising, but yet to take off. J Am Coll Cardiol. 2015;65:85-97. 
59. Singh SK, Desai ND, Petroff SD, Deb S, Cohen EA, Radhakrishnan S, et al. The impact of diabetic status on coronary artery bypass graft patency: insights from the radial artery patency study. Circulation. 2008;118:S222-5.

60. Deb S, Singh SK, Moussa F, Tsubota H, Une D, Kiss A, et al. The long-term impact of diabetes on graft patency after coronary artery bypass grafting surgery: a substudy of the multicenter Radial Artery Patency Study. J Thorac Cardiovasc Surg. 2014;148:1246-53.

61. Hoffman DM, Dimitrova KR, DeCastro H, Friedmann P, Geller CM, Ko W, et al. Improving long term outcome for diabetic patients undergoing surgical revascularization by use of the radial artery conduit: a propensity matched study. J Cardiothorac Surg. 2013;8:27.

62. Schwann TA, Al-Shaar L, Engoren M, Habib RH. Late effects of radial artery vs saphenous vein grafting for multivessel coronary bypass surgery in diabetics: a propensity-matched analysis. Eur J Cardiothorac Surg. 2013;44:701-10.

63. OECD. Health at a Glance 2015: OECD Indicators. Paris: OECD Publishing; 2015.

64. Deutscher Herzbericht 2014, DGK, DGTHG, DGPK, Deutsche Herzstiftung e.V. Publishing; Frankfurt am Main. In: 2016.

65. Beckmann A, Funkat AK, Lewandowski J, Frie M, Ernst M, Hekmat K, et al. Cardiac Surgery in Germany during 2014: a report on behalf of the German
Society for Thoracic and Cardiovascular Surgery. Thorac Cardiovasc Surg. 2015:63:258-69.

66. Patel MR, Dehmer GJ, Hirshfeld JW, Smith PK, Spertus JA. ACCF/SCAI/STS/ AATS/AHA/ASNC 2009 Appropriateness Criteria for Coronary Revascularization: a report by the American College of Cardiology Foundation Appropriateness Criteria Task Force, Society for Cardiovascular Angiography and Interventions, Society of Thoracic Surgeons, American Association for Thoracic Surgery, American Heart Association, and the American Society of Nuclear Cardiology Endorsed by the American Society of Echocardiography, the Heart Failure Society of America, and the Society of Cardiovascular Computed Tomography. J Am Coll Cardiol. 2009;53:530-53.

67. Kim LK, Feldman DN, Swaminathan RV, Minutello RM, Chanin J, Yang DC, et al. Rate of percutaneous coronary intervention for the management of acute coronary syndromes and stable coronary artery disease in the United States (2007 to 2011). Am J Cardiol. 2014;114:1003-10.

68. Desai NR, Bradley SM, Parzynski CS, Nallamothu BK, Chan PS, Spertus JA, et al. Appropriate Use Criteria for coronary revascularization and trends in utilization, patient selection, and appropriateness of percutaneous coronary intervention. JAMA. 2015;314:2045-53. 